\title{
VALUE DISTRIBUTION FOR THE GAUSS MAPS OF VARIOUS CLASSES OF SURFACES
}

\author{
YU KAWAKAMI \\ Dedicated to Professor Ryoichi Kobayashi on the occation of his sixtieth birthday
}

\begin{abstract}
We present in this article a survey of recent results in value distribution theory for the Gauss maps of several classes of immersed surfaces in space forms, for example, minimal surfaces in Euclidean $n$-space ( $n=3$ or 4 ), improper affine spheres in the affine 3 -space and flat surfaces in hyperbolic 3-space. In particular, we elucidate the geometric background of their results.
\end{abstract}

\section{INTRODUCTION}

The geometric nature of value distribution theory of complex analytic maps is well-known. One of the most notable results is the geometric interpretation of the precise maximum ' 2 ' for the number of exceptional values of a nonconstant meromorphic function on the complex plane C. Here we call a value that a function or map never attains an exceptional value of the function or map. In fact, Ahlfors [2] and Chern [7] proved that the least upper bound for the number of exceptional values of a nonconstant holomorphic map from $\mathbf{C}$ to a closed Riemann surface $\bar{\Sigma}_{\gamma}$ of genus $\gamma$ coincides with the Euler characteristic of $\bar{\Sigma}_{\gamma}$ by using Nevanlinna theory (see also [28, 42, 43, 44, 48]). In particular, for a nonconstant meromorphic function on $\mathbf{C}$, the geometric meaning of the maximal number ' 2 ' of exceptional values is the Euler characteristic of the Riemann sphere $\overline{\mathbf{C}}:=\mathbf{C} \cup\{\infty\}$. We remark that if the closed Riemann surface is of $\gamma \geq 2$, then such a map does not exist because the Euler characteristic is negative.

There exist several classes of immersed surfaces in 3-dimensional space forms whose Gauss maps have value-distribution-theoretic property. For instance, Fujimoto ([11, Theorem I], 14]) proved that the Gauss map of a nonflat complete minimal surface in Euclidean 3 -space $\mathbf{R}^{3}$ can omit at most 4 values. Moreover, Fujimoto [12] obtained a unicity theorem for the Gauss maps of nonflat complete minimal surfaces in $\mathbf{R}^{3}$, which is analogous to the Nevanlinna unicity theorem ([41]) for meromorphic functions on $\mathbf{C}$. On the other hand, the author and Nakajo 27. showed that the maximal number of exceptional values of the Lagrangian Gauss map of a weakly complete improper affine front in the affine 3 -space is 3 , unless it is an elliptic paraboloid. Moreover, the author [24] gave a similar result for flat fronts in hyperbolic 3-space $\mathbf{H}^{3}$.

The purpose of this review paper is to give geometric interpretation of value-distributiontheoretic property for their Gauss maps. The paper is organized as follows: In Section 2, we first give a curvature bound for the conformal metric $d s^{2}=\left(1+|g|^{2}\right)^{m}|\omega|^{2}$ on an open Riemann

2010 Mathematics Subject Classification. Primary 53A10; Secondary 30D35, 53C42.

Key words and phrases. Gauss map, Minimal surface, Front, Exceptional value, Unicity theorem .

Partly supported by the Grant-in-Aid for Scientific Research (C), No. 15K04840, Japan Society for the Promotion of Science. 
surface $\Sigma$, where $m$ is a positive integer, $\omega$ is a holomorphic 1-form and $g$ is a meromorphic function $g$ on $\Sigma$ (Theorems 2.1 and 2.2). As a corollary of it, we prove that the precise maximum for the number of exceptional values of $g$ on $\Sigma$ with the complete conformal metric $d s^{2}$ is ' $m+2$ ' (Corollary 2.3). We note that the geometric interpretation of the ' 2 ' in ' $m+2$ ' is the Euler characteristic of the Riemann sphere (Remark 2.5). We also give a unicity theorem (Theorem 2.6) for $g$ on $\Sigma$ with the complete metric $d s^{2}$. In Section 3, as application of our main results, we show some value-distribution-theoretic properties for the Gauss maps of the following classes of surfaces: minimal surfaces in $\mathbf{R}^{3}$ (Section 3.1), improper affine fronts in $\mathbf{R}^{3}$ (Section 3.2) and flat fronts in $\mathbf{H}^{3}$ (Section 3.3). Section 4 is denoted value-distribution-theoretic property for the Gauss map of a complete minimal surface in $\mathbf{R}^{4}$ (Section 4.1) and a complete minimal surface of finite total curvature in $\mathbf{R}^{3}$ (Section 4.2).

\section{MAin RESUlts}

We first give the following curvature bound for the conformal metric

$$
d s^{2}=\left(1+|g|^{2}\right)^{m}|\omega|^{2}
$$

on an open Riemann surface $\Sigma$.

Theorem 2.1 ([23]). Let $\Sigma$ be an open Riemann surface with the conformal metric

$$
d s^{2}=\left(1+|g|^{2}\right)^{m}|\omega|^{2},
$$

where $\omega$ is a holomorphic 1-from, $g$ is a meromorphic function on $\Sigma$, and $m$ is a positive integer. Assume that $g$ omits $q \geq m+3$ distinct values. Then there exists a positive constant $C$, depending on $m$ and the set of exceptional values, but not the surface, such that for all $p \in \Sigma$, we have

$$
\left|K_{d s^{2}}(p)\right|^{1 / 2} \leq \frac{C}{d(p)}
$$

where $K_{d s^{2}}(p)$ is the Gaussian curvature of $d s^{2}$ at $p$ and $d(p)$ is the geodesic distance from $p$ to the boundary of $\Sigma$, that is, the infimum of the lengths of the divergent curves in $\Sigma$ emanating from $p$.

More generally, when all of the multiple values of the meromorphic function $g$ in the metric (11) are totally ramified, the following theorem holds.

TheOrem $2.2([25])$. Let $\Sigma$ be an open Riemann surface with the conformal metric given by (11). Let $q$ be a positve integer, $\alpha_{1}, \ldots, \alpha_{q} \in \overline{\mathbf{C}}$ be distinct and $\nu_{1}, \ldots, \nu_{q} \in \mathbf{Z}_{+} \cup\{\infty\}$. Assume that

$$
\gamma:=\sum_{j=1}^{q}\left(1-\frac{1}{\nu_{j}}\right)>m+2 .
$$

If $g$ satisfies the property that all $\alpha_{j}$-points of $g$ have multiplicity at least $\nu_{j}$, then there exists a positive constant $C$, depending on $m, \gamma$ and $\alpha_{1}, \ldots, \alpha_{q}$ but not the surface, such that for all $p \in \Sigma$ inequality (2) holds. 
This is a generalization of Theorem 2.1. Indeed, we can show it by setting $\nu_{1}=\cdots=$ $\nu_{q=m+3}=\infty$. As an application of this theorem, we obtain an analogue of a special case of the Ahlfors islands theorem (See [4] for details of this theorem) for the meromorphic function $g$ on $\Sigma$ with the complete conformal metric $d s^{2}$. For more details, see [25].

As a corollary of these theorems, we give the least upper bound for the number of exceptional values of the meromorphic function $g$ on $\Sigma$ with the complete conformal metric given by (1).

Corollary 2.3 ([23]). Let $\Sigma$ be an open Riemann surface with the conformal metric given by (11). If the metric $d s^{2}$ is complete and the meromorphic function $g$ is nonconstant, then $g$ can omit at most $m+2$ distinct values.

Proof. By way of contradiction, suppose that $g$ omits $m+3$ distinct values. By Theorem 2.1. (11) holds. If $d s^{2}$ is complete, then we may set $d(p)=+\infty$ for all $p \in \Sigma$. Thus $K_{d s^{2}} \equiv 0$ on $\Sigma$. On the other hand, the Gaussian curvature with respect to the metric $d s^{2}$ is given by

$$
K_{d s^{2}}=-\frac{2 m\left|g_{z}^{\prime}\right|^{2}}{\left(1+|g|^{2}\right)^{m+2}\left|\hat{\omega}_{z}\right|^{2}},
$$

where $\omega=\hat{\omega}_{z} d z, g_{z}^{\prime}=d g / d z$. Thus $K_{d s^{2}} \equiv 0$ if and only if $g$ is constant. This contradicts the assumption that $g$ is nonconstant.

We give examples which ensure that Corollary 2.3 is optimal.

Proposition 2.4. Let $\Sigma$ be either the complex plane punctured at $q-1$ distinct points $\alpha_{1}, \ldots, \alpha_{q-1}$ or the universal cover of that punctured plane. We set

$$
\omega=\frac{d z}{\prod_{i=1}^{q-1}\left(z-\alpha_{i}\right)}, \quad g=z .
$$

Then $g$ omits $q$ distinct values and the metric $d s^{2}=\left(1+|g|^{2}\right)^{m}|\omega|^{2}$ is complete if and only if $q \leq m+2$. In particular, there exist examples whose metric $d s^{2}$ is complete and $g$ omits $m+2$ distinct values.

Proof. We can easily show that $g$ omits the $q$ distinct values $\alpha_{1}, \ldots, \alpha_{q-1}$ and $\infty$ on $\Sigma$. A divergent curve $\Gamma$ in $\Sigma$ must tend to one of the points $\alpha_{1}, \ldots, \alpha_{q-1}$ or $\infty$. Thus we have

$$
\int_{\Gamma} d s=\int_{\Gamma}\left(1+|g|^{2}\right)^{m / 2}|\omega|=\int_{\Gamma} \frac{\left(1+|z|^{2}\right)^{m / 2}}{\prod_{i=1}^{q-1}\left|z-\alpha_{i}\right|}|d z|=+\infty,
$$

when $q \leq m+2$.

REMARK 2.5. The geometric interpretation of the ' 2 ' in ' $m+2$ ' is the Euler characteristic of the Riemann sphere. Indeed, if $m=0$ then the metric $d s^{2}=\left(1+|g|^{2}\right)^{0}|\omega|^{2}=|\omega|^{2}$ is flat and complete on $\Sigma$. We thus may assume that $g$ is a meromorphic function on $\mathbf{C}$ because $g$ is replaced by $g \circ \pi$, where $\pi: \mathbf{C} \rightarrow \Sigma$ is a holomorphic universal covering map. On the other hand, Ahlfors [2] and Chern [7] showed that the best possible upper bound '2' of the number of exceptional values of nonconstant meromorphic functions on $\mathbf{C}$ coincides with the Euler characteristic of the Riemann sphere. Hence we get the conclusion. Remark that Ros [47] gave a different approach of this fact by using 'Bloch-Zalcman principle'. 
We next provide another type of value-distribution-theoretic property of the meromorphic function $g$ on an open Riemann surface $\Sigma$ with the conformal metric given by (1). Nevanlinna [41] showed that two nonconstant meromorphic functions on $\mathbf{C}$ having the same images for 5 distinct values must identically equal to each other. We obtain the following analogue to this unicity theorem.

THEOREM 2.6 ([25]). Let $\Sigma$ be an open Riemann surface with the conformal metric

$$
d s^{2}=\left(1+|g|^{2}\right)^{m}|\omega|^{2},
$$

and $\widehat{\Sigma}$ another open Riemann surface with the conformal metric

$$
d \hat{s}^{2}=\left(1+|\hat{g}|^{2}\right)^{m}|\hat{\omega}|^{2},
$$

where $\omega$ and $\hat{\omega}$ are holomorphic 1-forms, $g$ and $\hat{g}$ are nonconstant meromorphic functions on $\Sigma$ and $\widehat{\Sigma}$ respectively, and $m$ is a positive integer. We assume that there exists a conformal diffeomorphism $\Psi: \Sigma \rightarrow \widehat{\Sigma}$. Suppose that there exist $q$ distinct points $\alpha_{1}, \ldots, \alpha_{q} \in \overline{\mathbf{C}}$ such that $g^{-1}\left(\alpha_{j}\right)=(\hat{g} \circ \Psi)^{-1}\left(\alpha_{j}\right)(1 \leq j \leq q)$. If $q \geq m+5(=(m+4)+1)$ and either $d s^{2}$ or $d \hat{s}^{2}$ is complete, then $g \equiv \hat{g} \circ \Psi$.

We remark that Theorem 2.6 coincides with the Nevanlinna unicity theorem when $m=0$. The maps $g$ and $\hat{g} \circ \Psi$ are said to share the value $\alpha$ (ignoring multiplicity) when $g^{-1}(\alpha)=(\hat{g} \circ \Psi)^{-1}(\alpha)$. Theorem 2.6 is optimal for an arbitrary even number $m(\geq 2)$ because there exist the following examples.

Proposition $2.7([12,25])$. For an arbitrary even number $m(\geq 2)$, we take $m / 2$ distinct points $\alpha_{1}, \ldots, \alpha_{m / 2}$ in $\mathbf{C} \backslash\{0, \pm 1\}$. Let $\Sigma$ be either the complex plane punctured at $m+1$ distinct points $0, \alpha_{1}, \ldots, \alpha_{m / 2}, 1 / \alpha_{1}, \ldots, 1 / \alpha_{m / 2}$ or the universal covering of that punctured plane. We set

$$
\omega=\frac{d z}{z \prod_{i=1}^{m / 2}\left(z-\alpha_{i}\right)\left(\alpha_{i} z-1\right)}, \quad g(z)=z,
$$

and

$$
\hat{\omega}(=\omega)=\frac{d z}{z \prod_{i=1}^{m / 2}\left(z-\alpha_{i}\right)\left(\alpha_{i} z-1\right)}, \quad \hat{g}(z)=\frac{1}{z} .
$$

We can easily show that the identity map $\Psi: \Sigma \rightarrow \Sigma$ is a conformal diffeomorphism and the metrics $d s^{2}=\left(1+|g|^{2}\right)^{m}|\omega|^{2}$ is complete. Then the maps $g$ and $\hat{g}$ share the $m+4$ distinct values

$$
0, \infty, 1,-1, \alpha_{1}, \ldots, \alpha_{m / 2}, 1 / \alpha_{1}, \ldots, 1 / \alpha_{m / 2}
$$

and $g \not \equiv \hat{g} \circ \Psi$. These show that the number ' $m+5$ ' in Theorem 2.6 cannot be replaced by ' $m+4$ '.

\section{Applications}

In this section, as applications of the main results, we give some value-distribution-theoretic properties for the Gauss maps of several classes of surfaces. 
3.1. Gauss map of a complete minimal surface in $\mathbf{R}^{3}$. We first recall some basic facts of minimal surfaces in Euclidean 3-space $\mathbf{R}^{3}$. Details can be found, for example, in [13, 34] and [46]. Let $X=\left(x^{1}, x^{2}, x^{3}\right): \Sigma \rightarrow \mathbf{R}^{3}$ be an oriented minimal surface in $\mathbf{R}^{3}$. By associating a local complex coordinate $z=u+\sqrt{-1} v$ with each positive isothermal coordinate system $(u, v)$, $\Sigma$ is considered as a Riemann surface whose conformal metric is the induced metric $d s^{2}$ from $\mathbf{R}^{3}$. Then

$$
\triangle_{d s^{2}} X=0
$$

holds, that is, each coordinate function $x^{i}$ is harmonic. With respect to the local complex coordinate $z=u+\sqrt{-1} v$ of the surface, (6) is given by

$$
\bar{\partial} \partial X=0,
$$

where $\partial=(\partial / \partial u-\sqrt{-1} \partial / \partial v) / 2, \bar{\partial}=(\partial / \partial u+\sqrt{-1} \partial / \partial v) / 2$. Hence each $\phi_{i}:=\partial x^{i} d z(i=1,2,3)$ is a holomorphic 1 -form on $\Sigma$. If we set that

$$
\omega=\phi_{1}-\sqrt{-1} \phi_{2}, \quad g=\frac{\phi_{3}}{\phi_{1}-\sqrt{-1} \phi_{2}},
$$

then $\omega$ is a holomorphic 1-form and $g$ is a meromorphic function on $\Sigma$. Moreover the function $g$ coincides with the composition of the Gauss map and the stereographic projection from $\mathbf{S}^{2}$ onto $\overline{\mathbf{C}}$, and the induced metric is given by

$$
d s^{2}=\left(1+|g|^{2}\right)^{2}|\omega|^{2} .
$$

Applying Theorem 2.1 to the metric $d s^{2}$, we can obtain the Fujimoto curvature bound for a minimal surface in $\mathbf{R}^{3}$.

Theorem 3.1. [11, Theorem I and Corollary 3.4] Let $X: \Sigma \rightarrow \mathbf{R}^{3}$ be an oriented minimal surface whose Gauss map $g: \Sigma \rightarrow \overline{\mathbf{C}}$ omits greater than or equal to $5(=2+3)$ distinct values. Then there exists a positive constant $C$ depending on the set of exceptional values, but not the surface, such that for all $p \in \Sigma$ inequality (2) holds. In particular, the Gauss map of a nonflat complete minimal surface in $\mathbf{R}^{3}$ can omit at most $4(=2+2)$ values.

We note that this theorem is a generalization of the Bernstein theorem, stating that the only solution to the minimal surface equation over the whole plane is the trivial solution: a linear function $([3,6])$.

REMARK 3.2. For the Gauss maps of complete embedded minimal surfaces in $\mathbf{R}^{3}$, there exists an interesting conjecture called 'Four Point Conjecture'. For more details, see [37].

Moreover, by applying Theorem 2.6, we can get the Fujimoto unicity theorem for the Gauss maps of complete minimal surfaces in $\mathbf{R}^{3}$.

Theorem 3.3 ([13, Theorem I]). Let $X: \Sigma \rightarrow \mathbf{R}^{3}$ and $\widehat{X}: \widehat{\Sigma} \rightarrow \mathbf{R}^{3}$ be two nonflat minimal surfaces and assume that there exists a conformal diffeomorphism $\Psi: \Sigma \rightarrow \widehat{\Sigma}$. Let $g: \Sigma \rightarrow \overline{\mathbf{C}}$ and $\hat{g}: \widehat{\Sigma} \rightarrow \overline{\mathbf{C}}$ be the Gauss maps of $X(\Sigma)$ and $\widehat{X}(\widehat{\Sigma})$, respectively. If $g \not \equiv \hat{g} \circ \Psi$ and either $X(\Sigma)$ or $\widehat{X}(\widehat{\Sigma})$ is complete, then $g$ and $\hat{g} \circ \Psi$ share at most $6(=2+4)$ distinct values. 
3.2. Lagrangian Gauss map of a weakly complete improper affine front in $\mathbf{R}^{3}$. Improper affine spheres in the affine 3 -space $\mathbf{R}^{3}$ also have similar properties to minimal surfaces in Euclidean 3-space (for example, see [6]). Recently, Martínez [36] discovered the correspondence between improper affine spheres and smooth special Lagrangian immersions in the complex 2space $\mathbf{C}^{2}$ and introduced the notion of improper affine fronts, that is, a class of (locally strongly convex) improper affine spheres with some admissible singularities in $\mathbf{R}^{3}$. We note that this class is called 'improper affine maps' in [36, but we call this class 'improper affine fronts' because all of improper affine maps are wave fronts in $\mathbf{R}^{3}$ ([40], [51]). The differential geometry of wave fronts is discussed in [50]. Moreover, Martínez gave the following holomorphic representation for this class.

TheOrem 3.4 ([36, Theorem 3]). Let $\Sigma$ be a Riemann surface and $(F, G)$ a pair of holomorphic functions on $\Sigma$ such that $\operatorname{Re}(F d G)$ is exact and $|d F|^{2}+|d G|^{2}$ is positive definite. Then the induced map $\psi: \Sigma \rightarrow \mathbf{R}^{3}=\mathbf{C} \times \mathbf{R}$ given by

$$
\psi:=\left(G+\bar{F}, \frac{|G|^{2}-|F|^{2}}{2}+\operatorname{Re}\left(G F-2 \int F d G\right)\right)
$$

is an improper affine front. Conversely, any improper affine front is given in this way. Moreover we set $x:=G+\bar{F}$ and $n:=\bar{F}-G$. Then $L_{\psi}:=x+\sqrt{-1} n: \Sigma \rightarrow \mathbf{C}^{2}$ is a special Lagrangian immersion whose induced metric $d \tau^{2}$ from $\mathbf{C}^{2}$ is given by

$$
d \tau^{2}=2\left(|d F|^{2}+|d G|^{2}\right) .
$$

In addition, the affine metric $h$ of $\psi$ is expressed as $h:=|d G|^{2}-|d F|^{2}$ and the singular points of $\psi$ correspond to the points where $|d F|=|d G|$.

We remark that Nakajo [40] constructed a representation formula for indefinite improper affine spheres with some admissible singularities. The nontrivial part of the Gauss map of $L_{\psi}: \Sigma \rightarrow \mathbf{C}^{2} \simeq \mathbf{R}^{4}$ (see [9]) is the meromorphic function $\nu: \Sigma \rightarrow \overline{\mathbf{C}}$ given by

$$
\nu:=\frac{d F}{d G}
$$

which is called the Lagrangian Gauss map of $\psi$. An improper affine front is said to be weakly complete if the induced metric $d \tau^{2}$ is complete. We note that

$$
d \tau^{2}=2\left(|d F|^{2}+|d G|^{2}\right)=2\left(1+|\nu|^{2}\right)|d G|^{2} .
$$

Applying Theorem 2.1 to the metric $d \tau^{2}$, we can get the following theorem.

TheOREm 3.5 ([23, Theorem 4.6]). Let $\psi: \Sigma \rightarrow \mathbf{R}^{3}$ be an improper affine front whose Lagrangian Gauss map $\nu: \Sigma \rightarrow \overline{\mathbf{C}}$ omits greater than or equal to $4(=2+2)$ distinct values. Then there exists a positive constant $C$ depending on the set of exceptional values, but not $\Sigma$, such that for all $p \in \Sigma$ we have

$$
\left|K_{d \tau^{2}}(p)\right|^{1 / 2} \leq \frac{C}{d(p)}
$$

where $K_{d \tau^{2}}(p)$ is the Gaussian curvature of the metric $d \tau^{2}$ at $p$ and $d(p)$ is the geodesic distance from $p$ to the boundary of $\Sigma$. In particular, if the Lagrangian Gauss map of a weakly complete improper affine front in $\mathbf{R}^{3}$ is nonconstant, then it can omit at most $3(=1+2)$ values. 
Since the singular points of $\psi$ correspond to the points where $|\nu|=1$, we can obtain a simple proof of the parametric affine Bernstein theorem ([5], [21]) for improper affine spheres from the viewpoint of value-distribution-theoretic properties of the Lagrangian Gauss map.

Corollary $3.6\left([5,[21])\right.$. Any affine complete improper affine sphere in $\mathbf{R}^{3}$ must be an elliptic paraboloid.

Proof. Since an improper affine sphere has no singularities, the complement of the image of its Lagrangian Gauss map $\nu$ contains at least the circle $\{|\nu|=1\} \subset \overline{\mathbf{C}}$. Thus, by exchanging roles of $d F$ and $d G$ if necessarily, $|\nu|<1$ holds, that is, $|d F|<|d G|$. On the other hand, we have

$$
h=|d G|^{2}-|d F|^{2}<2\left(|d F|^{2}+|d G|^{2}\right)=d \tau^{2} .
$$

Thus if an improper affine sphere is affine complete, then it is also weakly complete. From Theorem 3.5 and [27, Proposition 3.1], it is an elliptic paraboloid.

By applying Theorem 2.6, we give the following unicity theorem for the Lagrangian Gauss maps of weakly complete improper affine fronts in $\mathbf{R}^{3}$.

Theorem 3.7 ([25, Theorem 4.24]). Let $\psi: \Sigma \rightarrow \mathbf{R}^{3}$ and $\widehat{\psi}: \widehat{\Sigma} \rightarrow \mathbf{R}^{3}$ be two improper affine fronts and assume that there exists a conformal diffeomorphism $\Psi: \Sigma \rightarrow \widehat{\Sigma}$. Let $\nu: \Sigma \rightarrow \overline{\mathbf{C}}$ and $\hat{\nu}: \widehat{\Sigma} \rightarrow \overline{\mathbf{C}}$ be the Lagrangian Gauss maps of $\psi(\Sigma)$ and $\widehat{\psi}(\widehat{\Sigma})$ respectively. Suppose that there exist $q$ distinct points $\alpha_{1}, \ldots, \alpha_{q} \in \overline{\mathbf{C}}$ such that $\nu^{-1}\left(\alpha_{j}\right)=(\hat{\nu} \circ \Psi)^{-1}\left(\alpha_{j}\right)(1 \leq j \leq q)$. If $q \geq 6(=(1+4)+1)$ and either $\psi(\Sigma)$ or $\widehat{\psi}(\widehat{\Sigma})$ is weakly complete, then either $\nu \equiv \hat{\nu} \circ \Psi$ or $\nu$ and $\hat{\nu}$ are both constant, that is, $\psi(\Sigma)$ and $\widehat{\psi}(\widehat{\Sigma})$ are both elliptic paraboloids.

3.3. Ratio of canonical forms of a weakly complete flat front in $\mathbf{H}^{3}$. For a holomorphic Legendrian immersion $\mathcal{L}: \Sigma \rightarrow S L(2, \mathbf{C})$ on a simply connected Riemann surface $\Sigma$, the projection

$$
f:=\mathcal{L} \mathcal{L}^{*}: \Sigma \rightarrow \mathbf{H}^{3}
$$

gives a flat front in $\mathbf{H}^{3}$. Here, flat fronts in $\mathbf{H}^{3}$ are flat surfaces in $\mathbf{H}^{3}$ with some admissible singularities (see [30], 33] for the definition of flat fronts in $\mathbf{H}^{3}$ ). We call $\mathcal{L}$ the holomorphic lift of $f$. Since $\mathcal{L}$ is a holomorphic Legendrian map, $\mathcal{L}^{-1} d \mathcal{L}$ is off-diagonal (see [16], [32], [33]). If we set

$$
\mathcal{L}^{-1} d \mathcal{L}=\left(\begin{array}{cc}
0 & \theta \\
\omega & 0
\end{array}\right),
$$

then the pull-back of the canonical Hermitian metric of $S L(2, \mathbf{C})$ by $\mathcal{L}$ is represented as

$$
d s_{\mathcal{L}}^{2}:=|\omega|^{2}+|\theta|^{2}
$$

for holomorphic 1-forms $\omega$ and $\theta$ on $\Sigma$. A flat front $f$ is said to be weakly complete if the metric $d s_{\mathcal{L}}^{2}$ is complete $([31,51])$. We define a meromorphic function on $\Sigma$ by the ratio of canonical forms

$$
\rho:=\frac{\theta}{\omega}
$$

Then a point $p \in \Sigma$ is a singular point of $f$ if and only if $|\rho(p)|=1$ ([29]). We note that

$$
d s_{\mathcal{L}}^{2}=|\omega|^{2}+|\theta|^{2}=\left(1+|\rho|^{2}\right)|\omega|^{2} .
$$


Applying Theorem 2.1 to the metric $d s_{\mathcal{L}}^{2}$, we can get the following theorem.

Theorem 3.8 ([23, Theorem 4.8]). Let $f: \Sigma \rightarrow \mathbf{H}^{3}$ be a flat front on a simply connected Riemann surface $\Sigma$. Suppose that the ratio of canonical forms $\rho: \Sigma \rightarrow \overline{\mathbf{C}}$ omits greater than or equal to $4(=2+2)$ distinct values. Then there exists a positive constant $C$ depending on the set of exceptional values, but not $\Sigma$, such that for all $p \in \Sigma$ we have

$$
\left|K_{d s_{\mathcal{L}}^{2}}(p)\right|^{1 / 2} \leq \frac{C}{d(p)}
$$

where $K_{d s_{\mathcal{L}}^{2}}(p)$ is the Gaussian curvature of the metric $d s_{\mathcal{L}}^{2}$ at $p$ and $d(p)$ is the geodesic distance from $p$ to the boundary of $\Sigma$. In particular, if the ratio of canonical forms of a weakly complete flat front in $\mathbf{H}^{3}$ is nonconstant, then it can omit at most $3(=1+2)$ values.

If $\Sigma$ is not simply connected, then we consider that $\rho$ is a meromorphic function on its universal covering surface $\widetilde{\Sigma}$. As an application of Theorem 3.8, we can obtain a simple proof of the classification of complete nonsingular flat surfaces in $\mathbf{H}^{3}$. For the proof, see [24, Corollary $3.5]$.

Corollary 3.9 ([49, 52]). Any complete nonsingular flat surface in $\mathbf{H}^{3}$ must be a horosphere or a hyperbolic cylinder.

Finally, by applying Theorem 2.6, we provide the following unicity theorem for the ratios of canonical forms of weakly complete flat fronts in $\mathbf{H}^{3}$.

Theorem 3.10 ([25, Theorem 4.29]). Let $f: \Sigma \rightarrow \mathbf{H}^{3}$ and $\widehat{f}: \widehat{\Sigma} \rightarrow \mathbf{R}^{3}$ be two flat fronts on simply connected Riemann surfaces and assume that there exists a conformal diffeomorphism $\Psi: \Sigma \rightarrow \widehat{\Sigma}$. Let $\rho: \Sigma \rightarrow \mathbf{C} \cup\{\infty\}$ and $\hat{\rho}: \widehat{\Sigma} \rightarrow \mathbf{C} \cup\{\infty\}$ be the ratio of canonical forms $f(\Sigma)$ and $\widehat{f}(\widehat{\Sigma})$ respectively. If $\rho \not \equiv \hat{\rho} \circ \Psi$ and either $f(\Sigma)$ or $f(\widetilde{\Sigma})$ is weakly complete, then $\rho$ and $\hat{\rho} \circ \Psi$ share at most $5=(1+4)$ distinct values.

REMARK 3.11. The hyperbolic Gauss map of a weakly complete or complete flat front in $\mathbf{H}^{3}$ has also interesting geometric property. For more details, see [29], 332, [33] and [39].

\section{FurTher TOPICS}

In this section, we give geometric interpretations of the maximal number of exceptional values and unicity theorem for the Gauss maps of complete minimal surfaces in $\mathbf{R}^{4}$. We also provide an effective estimate for the maximal number of exceptional values of the Gauss map of a nonflat complete minimal surface of finite total curvature in $\mathbf{R}^{3}$.

4.1. Gauss map of a complete minimal surface in $\mathbf{R}^{4}$. We first give an optimal estimate for the size of the image of the holomorphic map $G=\left(g_{1}, \ldots, g_{n}\right): \Sigma \rightarrow(\overline{\mathbf{C}})^{n}:=\underbrace{\overline{\mathbf{C}} \times \cdots \times \overline{\mathbf{C}}}_{n}$ on an open Riemann surface $\Sigma$ with the complete conformal metric

$$
d s^{2}=\prod_{i=1}^{n}\left(1+\left|g_{i}\right|^{2}\right)^{m_{i}}|\omega|^{2} .
$$


TheOrem 4.1 ([1, Theorem 2.1]). Let $\Sigma$ be an open Riemann surface with the conformal metric

$$
d s^{2}=\prod_{i=1}^{n}\left(1+\left|g_{i}\right|^{2}\right)^{m_{i}}|\omega|^{2},
$$

where $G=\left(g_{1}, \ldots, g_{n}\right): \Sigma \rightarrow(\overline{\mathbf{C}})^{n}$ is a holomorphic map, $\omega$ is a holomorphic 1-form on $\Sigma$ and each $m_{i}(i=1, \cdots, n)$ is a positive integer. Assume that $g_{i_{1}}, \ldots, g_{i_{k}}\left(1 \leq i_{1}<\cdots<i_{k} \leq n\right)$ are nonconstant and the others are constant. If the metric $d s^{2}$ is complete and each $g_{i_{l}}(l=1, \cdots, k)$ omits $q_{i_{l}}>2$ distinct values, then we have

$$
\sum_{l=1}^{k} \frac{m_{i_{l}}}{q_{i_{l}}-2} \geq 1 .
$$

We note that Theorem 4.1 also holds for the case where at least one of $m_{1}, \ldots, m_{n}$ is positive and the others are zeros. For instance, we assume that $g:=g_{i_{1}}$ is nonconstant and the others are constant. If $m:=m_{i_{1}}$ is a positive integer and the others are zeros, then inequality (9) coincides with

$$
\frac{m}{q-2} \geq 1 \Longleftrightarrow q \leq m+2,
$$

where $q:=q_{i_{1}}$. The result corresponds with Corollary 2.3.

We next give a unicity theorem for the holomorphic map $G=\left(g_{1}, \ldots, g_{n}\right): \Sigma \rightarrow(\overline{\mathbf{C}})^{n}$ on an open Riemann surface $\Sigma$ with the complete conformal metric defined by (9).

TheOREm 4.2 ([17, Theorem 2.1]). Let $\Sigma$ be an open Riemann surface with the conformal metric

$$
d s^{2}=\prod_{i=1}^{n}\left(1+\left|g_{i}\right|^{2}\right)^{m_{i}}|\omega|^{2}
$$

and $\widehat{\Sigma}$ another open Riemann surface with the conformal metric

$$
d \hat{s}^{2}=\prod_{i=1}^{n}\left(1+\left|\hat{g}_{i}\right|^{2}\right)^{m_{i}}|\hat{\omega}|^{2}
$$

where $\omega$ and $\hat{\omega}$ are holomorphic 1-forms, $G$ and $\widehat{G}$ are holomorphic maps into $(\overline{\mathbf{C}})^{n}$ on $\Sigma$ and $\widehat{\Sigma}$ respectively, and each $m_{i}(i=1, \cdots, n)$ is a positive integer. We assume that there exists a conformal diffeomorphism $\Psi: \Sigma \rightarrow \widehat{\Sigma}$, and $g_{i_{1}}, \ldots, g_{i_{k}}$ and $\hat{g}_{i_{1}}, \ldots, \hat{g}_{i_{k}}\left(1 \leq i_{1}<\cdots<i_{k} \leq n\right)$ are nonconstant and the others are constant. For each $i_{l}(l=1, \cdots, k)$, we suppose that $g_{i_{l}}$ and $\hat{g}_{i_{l}} \circ \Psi$ share $q_{i_{l}}>4$ distinct values and $g_{i_{l}} \not \equiv \hat{g}_{i_{l}} \circ \Psi$. If either $d s^{2}$ or $d \hat{s}^{2}$ is complete, then we have

$$
\sum_{l=1}^{k} \frac{m_{i_{l}}}{q_{i_{l}}-4} \geq 1 .
$$

We remark that Theorem 4.2 also holds for the case where at least one of $m_{1}, \ldots, m_{n}$ is positive and the others are zeros. For instance, we assume that $g:=g_{i_{1}}$ and $\hat{g}:=\hat{g}_{i_{1}}$ are nonconstant and the others are constant. If $m:=m_{i_{1}}$ is a positive integer and the others are zeros, then inequality (11) coincides with

$$
\frac{m}{q-4} \geq 1 \Longleftrightarrow q \leq m+4,
$$


where $q:=q_{i_{1}}$. The result corresponds with Theorem 2.6,

We will apply these results to the Gauss maps of complete minimal surfaces in $\mathbf{R}^{4}$. We briefly summarize here basic facts on minimal surfaces in $\mathbf{R}^{4}$. For more details, we refer the reader to [8, 18, 19, 45]. Let $X=\left(x^{1}, x^{2}, x^{3}, x^{4}\right): \Sigma \rightarrow \mathbf{R}^{4}$ be an oriented minimal surface in $\mathbf{R}^{4}$. By associating a local complex coordinate $z=u+\sqrt{-1} v$ with each positive isothermal coordinate system $(u, v), \Sigma$ is considered as a Riemann surface whose conformal metric is the induced metric $d s^{2}$ from $\mathbf{R}^{4}$. With respect to the local complex coordinate $z=u+\sqrt{-1} v$ of the surface, it holds that

$$
\bar{\partial} \partial X=0
$$

where $\partial=(\partial / \partial u-\sqrt{-1} \partial / \partial v) / 2, \bar{\partial}=(\partial / \partial u+\sqrt{-1} \partial / \partial v) / 2$. Hence each $\phi_{i}:=\partial x^{i} d z(i=$ $1,2,3,4)$ is a holomorphic 1 -form on $\Sigma$. If we set

$$
\omega=\phi_{1}-\sqrt{-1} \phi_{2}, \quad g_{1}=\frac{\phi_{3}+\sqrt{-1} \phi_{4}}{\phi_{1}-\sqrt{-1} \phi_{2}}, \quad g_{2}=\frac{-\phi_{3}+\sqrt{-1} \phi_{4}}{\phi_{1}-\sqrt{-1} \phi_{2}},
$$

then $\omega$ is a holomorphic 1-form, and $g_{1}$ and $g_{2}$ are meromorphic functions on $\Sigma$. Moreover the holomorphic map $G:=\left(g_{1}, g_{2}\right): \Sigma \rightarrow \overline{\mathbf{C}} \times \overline{\mathbf{C}}$ coincides with the Gauss map of $X(\Sigma)$. We remark that the Gauss map of $X(\Sigma)$ in $\mathbf{R}^{4}$ is the map from each point of $\Sigma$ to its oriented tangent plane, the set of all oriented (tangent) planes in $\mathbf{R}^{4}$ is naturally identified with the quadric

$$
\mathbf{Q}^{2}(\mathbf{C})=\left\{\left[w^{1}: w^{2}: w^{3}: w^{4}\right] \in \mathbf{P}^{3}(\mathbf{C}) ;\left(w^{1}\right)^{2}+\cdots+\left(w^{4}\right)^{2}=0\right\}
$$

in $\mathbf{P}^{3}(\mathbf{C})$, and $\mathbf{Q}^{2}(\mathbf{C})$ is biholomorphic to the product of the Riemann spheres $\overline{\mathbf{C}} \times \overline{\mathbf{C}}$. Furthermore the induced metric from $\mathbf{R}^{4}$ is given by

$$
d s^{2}=\left(1+\left|g_{1}\right|^{2}\right)\left(1+\left|g_{2}\right|^{2}\right)|\omega|^{2} .
$$

Applying Theorem 4.1 to the induced metric, we can obtain the Fujimoto theorem for the Gauss map of a complete minimal surface in $\mathbf{R}^{4}$.

ThEOREM 4.3. [11, Theorem II] Let $X: \Sigma \rightarrow \mathbf{R}^{4}$ be a complete nonflat minimal surface and $G=\left(g_{1}, g_{2}\right): \Sigma \rightarrow \overline{\mathbf{C}} \times \overline{\mathbf{C}}$ the Gauss map of $X(\Sigma)$.

(i) Assume that $g_{1}$ and $g_{2}$ are both nonconstant and omit $q_{1}$ and $q_{2}$ distinct values respectively. If $q_{1}>2$ and $q_{2}>2$, then we have

$$
\frac{1}{q_{1}-2}+\frac{1}{q_{2}-2} \geq 1
$$

(ii) If either $g_{1}$ or $g_{2}$, say $g_{2}$, is constant, then $g_{1}$ can omit at most 3 distinct values.

Proof. We first show (i). Since $g_{1}$ and $g_{2}$ are both nonconstant and $m_{1}=m_{2}=1$ from (12), we can prove inequality (13) by Theorem 4.1. Next we show (ii). If we set that $g_{1}$ omits $q_{1}$ values, then we obtain

$$
\frac{1}{q_{1}-2} \geq 1
$$

from Theorem 4.1 because $m_{1}=1$. Thus we have $q_{1} \leq 3$.

Hence we reveal that the Fujimoto theorem depends on the orders of the factors $\left(1+\left|g_{1}\right|^{2}\right)$ and $\left(1+\left|g_{2}\right|^{2}\right)$ in the induced metric from $\mathbf{R}^{4}$ and the Euler characteristic of the Riemann sphere $\overline{\mathbf{C}}$. In [1], we give some applications of this theorem, for example, to provide optimal results for 
the maximal number of exceptional values of the nontrivial part of the Gauss map of a complete minimal Lagrangian surface in the complex 2-space $\mathbf{C}^{2}$ and the generalized Gauss map of a complete nonorientable minimal surface in $\mathbf{R}^{4}$.

In the same way, by Theorem 4.2, we obtain a unicity theorem for the Gauss maps of complete minimal surfaces in $\mathbf{R}^{4}$.

Theorem 4.4. [17, Theorem 1.2] Let $X: \Sigma \rightarrow \mathbf{R}^{4}$ and $\widehat{X}: \widehat{\Sigma} \rightarrow \mathbf{R}^{4}$ be two nonflat minimal surfaces, and $G=\left(g_{1}, g_{2}\right): \Sigma \rightarrow \overline{\mathbf{C}} \times \overline{\mathbf{C}}, \widehat{G}=\left(\hat{g}_{1}, \hat{g}_{2}\right): \widehat{\Sigma} \rightarrow \overline{\mathbf{C}} \times \overline{\mathbf{C}}$ the Gauss maps of $X(\Sigma)$, $\widehat{X}(\widehat{\Sigma})$ respectively. We assume that there exists a conformal diffeomorphism $\Psi: \Sigma \rightarrow \widehat{\Sigma}$ and either $X(\Sigma)$ or $\widehat{X}(\widehat{\Sigma})$ is complete.

(i) Assume that $g_{1}, g_{2}, \hat{g}_{1}, \hat{g}_{2}$ are nonconstant and, for each $i(i=1,2), g_{i}$ and $\hat{g}_{i} \circ \Psi$ share $p_{i}>4$ distinct values. If $g_{1} \not \equiv \hat{g}_{1} \circ \Psi$ and $g_{2} \not \equiv \hat{g}_{2} \circ \Psi$, then we have

$$
\frac{1}{p_{1}-4}+\frac{1}{p_{2}-4} \geq 1 \text {. }
$$

In particular, if $p_{1} \geq 7$ and $p_{2} \geq 7$, then either $g_{1} \equiv \hat{g}_{1} \circ \Psi$ or $g_{2} \equiv \hat{g}_{2} \circ \Psi$, or both hold.

(ii) Assume that $g_{1}, \hat{g}_{1}$ are nonconstant, and $g_{1}$ and $\hat{g}_{1} \circ \Psi$ share $p$ distinct values. If $g_{1} \not \equiv$ $\hat{g}_{1} \circ \Psi$ and $g_{2} \equiv \hat{g}_{2} \circ \Psi$ is constant, then we have $p \leq 5$. In particular, if $p \geq 6$, then $G \equiv \widehat{G} \circ \Psi$.

4.2. Gauss map of a complete minimal surface of finite total curvature in $\mathbf{R}^{3}$. We review some of the standard facts on complete minimal surfaces of finite total curvature in $\mathbf{R}^{3}$. Let $X=\left(x^{1}, x^{2}, x^{3}\right): \Sigma \rightarrow \mathbf{R}^{3}$ be an oriented minimal surface in $\mathbf{R}^{3}$. Set $\phi_{i}:=\partial x^{i} d z$ $(i=1,2,3)$. These satisfy

(C) $\sum \phi_{i}^{2}=0$ : conformal condition,

(R) $\sum\left|\phi_{i}\right|^{2}>0$ : regularity condition,

(P) For every loop $\gamma \in H_{1}(\Sigma, \mathbf{Z}), \Re \int_{\gamma} \phi_{i}=0$ : period condition.

For the meromorphic function $g$ and holomorphic 1-form $\omega$ given by (17),

$$
\phi_{1}=\frac{1}{2}\left(1-g^{2}\right) \omega, \quad \phi_{2}=\frac{\sqrt{-1}}{2}\left(1+g^{2}\right) \omega, \quad \phi_{3}=g \omega
$$

hold. We call $(\omega, g)$ the Weierstrass data (W-data, for short). If we are given the W-data on $\Sigma$, we get $\phi_{j}$ 's by this formula. They satisfy condition (C) automatically, and condition (R) is interpreted as the poles of $g$ of order $l$ coincides exactly with the zeros of $\omega$ of order $2 k$, because the induced metric $d s^{2}$ is given by (8). In general, for a given meromorphic function $g$ on $\Sigma$, it is not so hard to find a holomorphic 1-form $\omega$ satisfying condition (R). However, the period condition (P) always causes trouble. The total curvature of $X(\Sigma)$ is given by

$$
\tau(\Sigma):=\int_{\Sigma} K_{d s^{2}} d A=-\int_{\Sigma} \frac{2 \sqrt{-1} d g \wedge d \bar{g}}{\left(1+|g|^{2}\right)^{2}},
$$

where $d A$ is the area element with respect to the metric $d s^{2}$. Note that $|\tau(\Sigma)|$ is the area of $\Sigma$ with respect to the metric induced from the Fubini-Study metric of the Riemann sphere $\overline{\mathbf{C}}$ by $g$.

TheOREM 4.5. A complete minimal surface of finite total curvature $X: \Sigma \rightarrow \mathbf{R}^{3}$ satisfies 
(i) $\Sigma$ is conformally to $\bar{\Sigma}_{\gamma} \backslash\left\{p_{1}, \ldots, p_{k}\right\}$, where $\bar{\Sigma}_{\gamma}$ is a closed Riemann surface of genus $\gamma$ and $p_{1}, \ldots, p_{k} \in \bar{\Sigma}([20])$,

(ii) The $W$-date $(\omega, g)$ can be extended meromorphically to $\bar{\Sigma}_{\gamma}([45])$.

From this fact, we call such surfaces algebraic minimal surfaces. Osserman proved the following result for the number of exceptional values of the Gauss map of a complete minimal surface of finite total curvature in $\mathbf{R}^{3}$.

Theorem 4.6. [45, Theorem 3] The Gauss map of a nonflat complete minimal surface of finite total curvature omits at most 3 values.

The author, Kobayashi and Miyaoka refined Theorem 4.6 and give the following estimate for the number of exceptional values of the Gauss map of a complete minimal surface of finite total curvature in $\mathbf{R}^{3}$.

Theorem 4.7. [26, Theorem 3.3] Let $X: \Sigma=\bar{\Sigma}_{\gamma} \backslash\left\{p_{1}, \ldots, p_{k}\right\} \rightarrow \mathbf{R}^{3}$ be a nonflat complete minimal surface of finite total curvature, $g: \Sigma \rightarrow \overline{\mathbf{C}}$ its Gauss map, $d$ the degree of $g$ considered as a map on $\bar{\Sigma}_{\gamma}$. Then the number $D_{g}$ of exceptinal values of $g$ satisfies

$$
D_{g} \leq 2+\frac{2}{R}, \quad R=\frac{d}{\gamma-1+(k / 2)}>1 .
$$

ProOF. By a suitable rotation of the surface in $\mathbf{R}^{3}$, we may assume that the Gauss map $g$ has neither zero nor pole at $p_{j}$ and that the zeros and poles of $g$ are simple. The simple poles of $g$ coincide with the double zeros of $\omega$ because the surface satifies the regularity condition (R). By the completeness of the surface, $\omega$ has a pole at each end $p_{j}$ ([35], [46, Lemma 9.6]). Moreover, since the surface satisfies the period condition $(\mathrm{P}), \omega$ has a pole of order $\mu_{j} \geq 2$ at $p_{j}$ ([45]). Applying the Riemann-Roch theorem to $\omega$ on $\bar{\Sigma}_{\gamma}$, we obtain that

$$
2 d-\sum_{j=1}^{k} \mu_{j}=2 \gamma-2 \text {. }
$$

Thus we have

$$
d=\gamma-1+\frac{1}{2} \sum_{j=1}^{k} \mu_{j} \geq \gamma-1+k>\gamma-1+(k / 2),
$$

and $R>1$.

On the other hand, we assume that $g$ omits $D_{g}$ values. Let $n_{0}$ be the sum of the branching orders at the image of exceptional values. Then we have

$$
k \geq d D_{g}-n_{0} .
$$

Let $n_{g}$ be the total branching order of $g$ on $\bar{\Sigma}_{\gamma}$. Then applying the Riemann-Hurwitz formula to the meromorphic function $g$ on $\bar{\Sigma}_{\gamma}$, we have

$$
n_{g}=2(d+\gamma-1) \text {. }
$$

Hence we have

$$
D_{g} \leq \frac{n_{0}+k}{d} \leq \frac{n_{g}+k}{d}=2+\frac{2}{R}
$$


REMARK 4.8. More precisely, (15) holds for the totally ramified value number $\nu_{g}$ for the Gauss map of a complete minimal surface of finite total curvature in $\mathbf{R}^{3}$. One of the most important results for the number is to discover nonflat complete minimal surfaces of finite total curvature in $\mathbf{R}^{3}$ with $\nu_{g}=2.5$. For the details, see [22].

By the proof of Theorem 4.7, we reveal that the reason why the upper bound for $D_{g}$ changes from ' 4 ' to ' 3 ' is that the order $\mu_{j}$ of a pole of $\omega$ at each end $p_{j}$ changes from ' $\mu_{j} \geq 1$ ' to ' $\mu_{j} \geq 2$ '. Remark that this principle is equivalent to the distinction between the Cohn-Vossen inequality and the Osserman inequality on the total curvature of a complete minimal surface of finite total curvature in $\mathbf{R}^{3}$.

There still remains the following question.

Problem 4.9 ([45]). Does there exist a complete minimal surface of finite total curvature in $\mathbf{R}^{3}$ whose Gauss map omits 3 values?

If so, Theorems 4.6 and 4.7 are optimal. If not, the maximum is ' 2 ' and is attained by the catenoid and examples constructed by Miyaoka and Sato [38. In regards to this problem, the following facts are well-known.

Proposition 4.10. For a nonflat complete minimal surface of finite total curvature in $\mathbf{R}^{3}$,

(i) When $\gamma=0$, the Gauss map omits at most 2 values ([45], [26]),

(ii) When $\gamma=1$ and the surface has a non-embedded end, the Gauss map omits at most 2 values ([15], [10], 26]),

(iii) If the Gauss map omits 3 values, then $\gamma \geq 1$ and the total curvature $\tau(\Sigma) \leq-20 \pi([45$, [53, [10]).

By virtue of Theorem 4.7 and this proposition, if there exists a complete minimal surface of finite total curvature whose Gauss map omits 3 values, then it has the complexity of topological data. Thus it is very hard to solve the period condition $(\mathrm{P})$ of the surface. This is the difficulty of this problem.

\section{REFERENCES}

[1] R. Aiyama, K. Akutagawa, S. Imagawa and Y. Kawakami, Remarks on the Gauss images of complete minimal surfaces in Euclidean four-space, to appear in Annali di Matematica Pura ed Applicata, (DOI: 10.1007/s10231-017-0643-6).

[2] L. V. Ahlfors, Zur Theorie der Überlagerungsflächen, Acta Math., 65 (1935) 157-194, and Collected Papers Vol. I, pp. 163-173.

[3] S. Bernstein, Sur un théorème de géométrie et ses applications aux équations aux dérivées partielles du type elliptique, Comm. de la Soc. Math. de Kharkov (2éme sér.) 15, 38-45 (1915-1917).

[4] W. Bergweiler, The role of the Ahlfors five islands theorem in complex dynamics, Conform. Geom. Dyn., 4 (2000), 22-34.

[5] E. Calabi, Improper affine hypersurfaces of convex type and a generalization of a theorem by K. Jörgens, Mich. Math. J., 5 (1958), 108-126.

[6] E. Calabi, Examples of Bernstein problems for some nonlinear equations, In: Global Analysis, Berkeley, CA, 1968 (eds. S.-S. Chern and S. Smale), Proc. Sympos. Pure Math., 15, Amer. Math. Soc. Providence, RI, pp. $223-230$.

[7] S.-S. Chern, Complex analytic mappings of Riemann surfaces. I, Amer. J. Math., 82 (1960), 323-337. 
[8] S. S. Chern, Minimal surfaces in an Euclidean space of $N$ dimensions, 1965 Differential and Combinatorial Topology (A Symposium in Honor of Marston Morse), 187-198, Princeton Univ. Press, Princeton, N.J.

[9] B.-Y. Chen and J.-M. Morvan, Géométrie des surfaces lagrangiennes de C ${ }^{2}$, J. Math. Pures Appl., 66 (1987), 321-325.

[10] Y. Fang, On the Gauss map of complete minimal surfaces with finite total curvature, Indiana Univ. Math. J., 42 (1993), 1389-1411.

[11] H. Fujimoto, On the number of exceptional values of the Gauss map of minimal surfaces, J. Math. Soc. Japan, 40 (1988), 237-249.

[12] H. Fujimoto, Unicity theorems for the Gauss maps of complete minimal surfaces, J. Math. Soc. Japan, 45 (1993), 481-487.

[13] H. Fujimoto, Value distribution theory of the Gauss map of minimal surfaces in $\mathbf{R}^{m}$, Aspects of Mathematics, E21. Friedr. Vieweg \& Sohn, Braunschweig, 1993.

[14] H. Fujimoto, Nevanlinna theory and minimal surfaces, In: Geometry V: Minimal Surfaces (ed. R. Osserman), Encyclopaedia Math. Sci., 90, Springer, Berlin, 1997, pp. 95-151; pp. 267-272.

[15] F. Gackstätter, Über abelsche Minimalflächen, Math. Nachr., 74 (1976), 165-165.

[16] J. A. Gálvez, A. Martínez and F. Milán, Flat surfaces in hyperbolic 3-space, Math. Ann., 316 (2000), $419-435$.

[17] P. H. Ha and Y. Kawakami, A note on a unicity theorem for the Gauss maps of complete minimal surfaces in Euclidean four-space, to appear in Canadian Mathematical Bulletin, (DOI: 10.4153/CMB-2017-015-0).

[18] D. A. Hoffman and R. Osserman, The geometry of the generalized Gauss map, Mem. Amer. Math. Soc. 28 (1980), no. 236.

[19] D. A. Hoffman and R. Osserman, The Gauss map of surfaces in $\mathbf{R}^{3}$ and $\mathbf{R}^{4}$, Proc. London Math. Soc., 50 (1985), 27-56.

[20] A. Huber, On subharmonic functions and differential geometry in the large, Comment. Math. Helv., 32 (1957), 13-72.

[21] K. Jörgens, Über die Lösungen der differentialgleichung $r t-s^{2}=1$, Math. Ann., 127 (1954), $130-134$.

[22] Y. Kawakami, On the totally ramified value number of the Gauss map of minimal surfaces, Proc. Japan Acad. Ser. A Math. Sci., 82 (2006), 1-3.

[23] Y. Kawakami, On the maximal number of exceptional values of Gauss maps for various classes of surfaces, Math. Z., 274 (2013), 1249-1260.

[24] Y. Kawakami, A ramification theorem for the ratio of canonical forms of flat surfaces in hyperbolic threespace, Geom. Dedicata, 171 (2014), 387-396.

[25] Y. Kawakami, Function-theoretic properties for the Gauss maps of various classes of surfaces, Canad. J. Math., 67 (2015), 1411-1434.

[26] Y. Kawakami, R. Kobayashi and R. Miyaoka, The Gauss map of pseudo-algebraic minimal surfaces, Forum Math., 20 (2008), 1055-1069.

[27] Y. Kawakami and D. Nakajo, Value distribution of the Gauss map of improper affine spheres, J. Math. Soc. Japan, 64 (2012), 799-821.

[28] R. Kobayashi, Toward Nevanlinna theory as a geometric model for Diophantine approximation, Sugaku Expositions, 16 (2003), 39-79.

[29] M. Kokubu, W. Rossman, K. Saji, M. Umehara and K. Yamada, Singularities of flat fronts in hyperbolic space, Pacific J. Math., 221 (2005), 303-351.

[30] M. Kokubu, W. Rossman, M. Umehara and K. Yamada, Flat fronts in hyperbolic 3-space and their caustics, J. Math. Soc. Japan, 59 (2007), 265-299.

[31] M. Kokubu, W. Rossman, M. Umehara and K. Yamada, Asymptotic behavior of flat surfaces in hyperbolic 3-space, J. Math. Soc. Japan, 61 (2009), 799-852.

[32] M. Kokubu, M. Umehara and K. Yamada, An elementary proof of Small's formula for null curves in PSL(2, C) and an analogue for Legendrian curves in PSL(2, C), Osaka J. Math. 40 (2003), 697-715.

[33] M. Kokubu, M. Umehara and K. Yamada, Flat fronts in hyperbolic 3-space, Pacific J. Math., 216 (2004), 149-175. 
[34] H. B. Lawson Jr., Lectures on minimal submanifolds, Vol. I, Mathematics Lecture Series 9, Publish or Perish, Inc., 1980.

[35] G. R. Maclane, On asymptotic values, abstract 603-166, Notices Amer. Math. Soc., 10 (1963), $482-483$.

[36] A. Martínez, Improper affine maps, Math. Z., 249 (2005), 755-766.

[37] W. H. Meeks III and J. Pérez, A survey on classical minimal surface theory. University Lecture Series, 60, American Mathematical Society, Providence, RI, 2012.

[38] R. Miyaoka and K. Sato, On complete minimal surfaces whose Gauss map misses two directions, Arch. Math., 63 (1994), 565-576.

[39] F. Matín, M. Umehara and K. Yamada, Flat surfaces in hyperbolic 3-space whose hyperbolic Gauss maps are bounded, Rev. Mat. Iberoam., 30 (2014), 309-316.

[40] D. Nakajo, A representation formula for indefinite improper affine spheres, Results Math., 55 (2009), 139-159.

[41] R. Nevanlinna, Einige Eindeutigkeitssätze in der Theorie der Meromorphen Funktionen, Acta Math., 48 (1926), 367-391.

[42] R. Nevanlinna, Analytic functions, Translated from the second German edition by Phillip Emig. Die Grundlehren der mathematischen Wissenschaften, Band 162, Springer-Verlag, New York-Berlin, 1970.

[43] J. Noguchi and T. Ochiai, Geometric function theory in several complex variables, Transl. Math. Monogt. 80, Amer. Math. Soc., Providence, RI, 1990.

[44] J. Noguchi and J. Winkelmann, Nevanlinna theory in several complex variables and Diophantine approximation, Grundlehren der mathematischen Wissenschaften, Vol. 350, Springer, 2014.

[45] R. Osserman, Global properties of minimal surfaces in $E^{3}$ and $E^{n}$, Ann. of Math., 80 (1964), $340-364$.

[46] R. Osserman, A survey of minimal surfaces, 2nd edition, Dover Publication Inc., New York, 1986.

[47] A. Ros, The Gauss map of minimal surfaces, Differential geometry, Valencia, 2001, 235-252, World Sci. Publ., 2002.

[48] M. Ru, Nevanlinna theory and its relation to Diophantine approximation, World Sci., River Edge, NJ, 2001.

[49] S. Sasaki, On complete flat surfaces in hyperbolic 3-space, Kōdai Math Sem. Rep., 25 (1973), $449-457$.

[50] K. Saji, M. Umehara and K. Yamada, The geometry of fronts, Ann. of Math., 169 (2009), 491-529.

[51] M. Umehara and K. Yamada, Applications of a completeness lemma in minimal surface theory to various classes of surfaces, Bull. London Math. Soc., 43 (2011), 191-199. Corrigendum, Bull. London Math. Soc., 44 (2012), 617-618.

[52] Y. A. Volkov and S. M. Vladimirova, Isometric immersions of the Euclidean plane in Lobačevskii space (Russian), Mat. Zametki, 10 (1971), 327-332.

[53] A. Weitsman and F. Xavier, Some function theoretic properties of the Gauss map for hyperbolic complete minimal surfaces, Mich. Math. J., 34 (1987), 275-283.

Faculty of Mathematics And Physics, Institute of Science And EngineERIng, KANAZAWA UNIVERSITY, KANAZAWA, 920-1192, JAPAN

E-mail address: y-kwkami@se.kanazawa-u.ac.jp 